\title{
Retour sur le désencastrement
}

Polanyi ou la science économique vue comme une institution influençant l'évolution des systèmes économiques

Nicolas Brisset

\section{OpenEdition}

1 Journals

Édition électronique

URL : http://journals.openedition.org/ress/1080

DOI : $10.4000 /$ ress. 1080

ISSN : 1663-4446

Éditeur

Librairie Droz

Édition imprimée

Date de publication : 15 juin 2012

Pagination : 7-39

ISBN : 978-2-600-01613-1

ISSN : 0048-8046

Référence électronique

Nicolas Brisset, «Retour sur le désencastrement », Revue européenne des sciences sociales [En ligne], 50-1 | 2012, mis en ligne le 15 juin 2015, consulté le 10 décembre 2020. URL : http://

journals.openedition.org/ress/1080 ; DOI : https://doi.org/10.4000/ress.1080 


\title{
RETOUR SUR LE DÉSENCASTREMENT \\ POLANYI OU LA SCIENCE ÉCONOMIQUE VUE COMME UNE INSTITUTION INFLUENÇANT L'ÉVOLUTION DES SYSTÈMES ÉCONOMIQUES
}

NICOLAS BRISSET

Université de Lausanne - Centre d'études interdisciplinaires Walras-Pareto / Université de Paris-I - Centre d'économie de la Sorbonne nicolas.brisset@unil.ch

\begin{abstract}
Résumé. L'ambition de cet article est de montrer qu'un regard original porté sur le magnum opus de Karl Polanyi, La Grande Transformation, ouvre de nouvelles perspectives dans la portée analytique de celui-ci, nuançant par là même les critiques auxquelles il prête le flanc, principalement axées autour de la notion de désencastrement. Pour cela, il tentera de réinterpréter ce débat en termes épistémologiques, i.e. de lien entre concept et réalité, et par la suite de rapprocher Karl Polanyi de la notion de performativité des énoncés, aujourd'hui fleurissante dans le domaine de la sociologie économique.
\end{abstract}

Mots-clés: Karl Polanyi, performativité, désencastrement, réalisme.

\begin{abstract}
Our ambition is to point out that a fresh look at what is usualy considered to be Karl Polanyi's magnum opus, The Great Transformation, opens some new prospects on its analytical. This interpretation qualifies some of the Hungarian author's main critics, directed against his concept of desembeddedness. With this aim in mind, we re-interpret this debate in epistemological terms, i.e. the link between concepts and reality ; then we try to connect Karl Polanyi with the notion of performativity, which is today flourishing, above all within the field of economic sociology.
\end{abstract}

Keywords: Karl Polanyi, performativity, desembeddedness, realism.

Code JEL: B25, B4I, ZI3. 
Choisissez, par exemple, une motivation quelconque, et organisez la production de manière qu'elle incite effectivement les individus à produire; vous aurez, en même temps, créé une image de l'Homme telle qu'il semblera totalement réduit à cette seule motivation. [.. .] La motivation choisie dessinera la figure de l’Homme réel. Polanyi, I977a, p. I8

\section{INTRODUCTION}

La notoriété de Karl Polanyi doit beaucoup à La Grande Transformation [GT dans la suite du texte]. Cet ouvrage a suscité un certain nombre de débats, dont un a particulièrement marqué l'histoire des idées économiques : celui opposant deux interprétations du concept de désencastrement. Dans son livre, Polanyi reconstruit l'histoire, au XIX ${ }^{\mathrm{e}}$ siècle, de la tentative de mise en place d'une société de marché, venant soutenir un système inédit de coordination des échanges : un grand marché autorégulateur. Un tel système aurait eu comme caractéristique un désencastrement progressif de l'économie vis-à-vis des structures sociales traditionnelles: «la société est gérée en tant qu’auxiliaire du marché. Au lieu que l'économie soit encastrée dans les relations sociales, ce sont les relations sociales qui sont encastrées dans le système économique » (Polanyi, 1944, p. 88). C'est ainsi, par exemple, que l'on transforme peu à peu le travail en marchandise en l'autonomisant des solidarités traditionnelles afin de constituer une large offre de travail venant alimenter un marché du travail.

Selon certains tenants de la nouvelle sociologie économique, plus particulièrement Granovetter (1985), le concept de désencastrement serait porteur d'une ambiguïté insurmontable. En effet, celui-ci amènerait à penser le monde économique hors des institutions sociales, vision vivement critiquée par Granovetter, pour qui il n'est tout simplement pas possible de penser l'économie en dehors d'une architecture institutionnelle dont le pan économique ne serait qu'une partie. Cette vision est d’ailleurs également le fait d'un courant auquel on associe souvent Polanyi lui-même (Chavance, 2007), à savoir l’ancien institutionnalisme américain. 
Polanyi donnerait de l'acteur économique une vision sous-socialisée là où la sociologie économique et l'institutionnalisme s'attacheraient au contraire à enrichir les visions, jugées simplistes, de l’agent économique. Le désencastrement est ici saisi comme la fin des institutions. À l'encontre de cette position, on trouve un certain nombre de commentateurs optant pour une vision avant tout idéologique du désencastrement (Maucourant, 2005, p. 92-95; Le Velly, 2007 ; Plociniczack, 2007 ; Block, 2003) : l'économie désencastrée n’est pas un état de fait mais une utopie en marche, utopie consistant à croire que l'ensemble des problèmes relatifs à la subsistance sont solubles dans les mécanismes du marché.

L’objectif de cet article sera de montrer qu'une nouvelle interprétation de la GT ouvre de nouvelles perspectives dans sa portée analytique et nuance les critiques auxquelles elle prête le flanc, ceci en venant appuyer la vision idéologique du désencatrement. On utilisera pour cela la notion de performativité des énoncés qui, appliquée à la science économique, prend son origine dans l'ouvrage collectif, dirigé par Michel Callon The Law of the Market (Callon, I998), et retrouve aujourd'hui une certaine vigueur avec la parution d'un nouvel ouvrage collectif, Do Economists Make Market? (MacKenzie, Muniesa et Siu, 2007). La performativité désigne l'idée que la science en général, l'économie en ce qui nous concerne ici, sculpte et forme la réalité économique plutôt qu’elle ne décrit la manière dont elle fonctionne ${ }^{1}$. C'est dans une telle perspective que la thèse de Polanyi sera appréhendée.

Muni d'une telle interprétation, ce travail tentera de montrer que non seulement Karl Polanyi trouve une place au côté des institutionnalistes, mais également que son institutionnalisme est d'un type particulier en ce qu'il confère à la science économique un statut spécifique: celui d'une institu-

I La problématique de la performativité des énoncés nous vient des travaux du linguiste John Langshaw Austin (1962). Son projet originel était d'ouvrir la voie à une distinction entre énoncés constatifs, qui ne font que constater un état du monde, et énoncés performatifs, qui, en eux-mêmes, constituent un acte influençant l'état du monde. Par exemple: «'oui [je le veux]' [... 'je baptise ce bateau le Queen Elizabeth' [...], 'Je vous parie six pence qu'il pleuvra demain» (Austin, 1962, p.4I). Ne sera pas abordée ici la migration de ce concept vers le champ des sciences sociales. Pour un point de vue général, voir Denis, 2006. Sur les débats relatifs à la performativité, voir Brisset, 2011 . 
tion ayant une place majeure dans l'évolution des systèmes économiques. Ce travail montrera que le mécanisme de désencastrement peut être vu, non comme la réalisation effective d'une économie désencastrée, mais comme l'influence d'une certaine théorie économique sur la réalité économique et sociale. Dans le cadre de la GT, ce sont les concepts de la théorie économique classique qui viennent légitimer une structure institutionnelle en place par le biais d'un discours naturaliste. Ainsi la théorie entraîne un phénomène de blocage de l'évolution des institutions.

Pour asseoir une telle argumentation, il faudra avant tout réinterpréter le problème du désencastrement en termes épistémologiques. On peut en effet noter que, en dernière analyse, la sociologie économique pointe du doigt la possibilité, chez Polanyi, de la réalisation d'un concept théorique, celui d'«économie désencastrée ».

La première partie sera l'occasion de revenir sur la critique de l'économie politique développée par Polanyi et sur la base de laquelle il est amené à produire un certain nombre d'outils analytiques qui serviront l'historiographie de la GT. Ce n'est qu'une fois ce travail effectué qu'il sera possible, dans un second temps, de souligner les problèmes épistémologiques sous-jacents au problème du désencastrement, problèmes que l'on sera en mesure de résoudre via une lecture en termes de performativité des énoncés. Réinterpréter ainsi Karl Polanyi nécessitera une reconstruction de sa pensée. Si la GT est avant tout organisée comme un récit historique, cette approche narrative est abordée en cohabitation avec un modèle action-structure sous-jacent ${ }^{2}$.

2 Revenant sur le lien entre narration et modélisation, Dumez et Jeunemaître en arrivent à la conclusion suivante: «En réalité, l'approche narrative n'est pas traitée en elle-même, comme un récit approfondi, déployant les faits, explorant des hypothèses rivales, mais comme un élément ancillaire de la modélisation destiné à en assurer la plausibilité» (Dumez et Jeunemaître, 2005, p.998). L'interprétation ici proposée de l'œuvre de Polanyi se situe dans une optique similaire, aujourd'hui nommée narration analytique. 


\section{L'ÉCONOMIE COMME PROCESSUS INSTITUTIONNALISÉ Polanyi ou l'impossibilité logique du désencastrement}

L'objectif premier de la GT, récurrent dans l'œuvre de Polanyi, est de comprendre l'histoire économique et sociale au vu de grands principes d'organisation sociétale. Ces principes laissent entrevoir une épistémologie et une méthodologie particulières, que l'on peut à première vue qualifier d'institutionnalistes. Néanmoins, c'est au regard de ces points précis que l'historiographie polanyienne semble porteuse d'une argumentation contradictoire. Dans un premier temps sera exposé le projet polanyien. Ce n'est que dans un second temps que sera dégagé ce qui semble être un problème de cohérence au sein de l'œuvre. Ceci conduira à développer un élément interprétatif comme réponse à ce paradoxe, élément qui sera utilisé dans la seconde partie de ce travail.

\section{I.I.ÉCONOMIE FORMELLE VS ÉCONOMIE SUBSTANTIVE}

Dans la GT, ainsi que dans un article intitulé «L'économie en tant que procès institutionalisé» (1957a), Polanyi s’attaque en premier lieu à la théorie économique telle qu'elle est alors pratiquée, i.e. concentrée uniquement et de façon obsessionnelle sur «le prix comme étant le fait économique par excellence», décrivant ainsi «l'économie toute entière comme déterminée par les choix suscités par la rareté des moyens» (Polanyi, 1957a, p. 55). Ce type de théorie économique est dit «formel». De manière générale, cette définition de l'économie renvoie à celle qu'en donne Robbins dans son célèbre An Essay on the Nature and Significance of Economic Science ${ }^{3}$. Lobjet de l'économie est ainsi réduit au choix sous contrainte.

Polanyi veut, lui, s'engager dans une voie différente, celle de l'économie substantive. Ce nouveau sens se réfère à l'économie en tant que processus institutionnalisé, i.e. comme un ensemble d'interactions constantes entre l'homme, agissant dans la quête de sa subsistance, et son environnement physique et social. Deux notions sont convoquées : celle de processus et celle d'institution. La première fait référence à une analyse en termes de mouvements matériels 
englobant la production, la mise à disposition et l'appropriation des biens. La seconde au fait que ces mouvements prennent forme au sein d'une structure d'interactions relevant de la coutume, de la loi, autrement dit d'un appareillage institutionnel. D’où le caractère institutionnalisé du processus ${ }^{4}$.

La principale critique de Polanyi envers l'économie formelle est que lorsque celle-ci entreprend d'étudier les économies particulières par le truchement du principe «universel» du choix, elle néglige la nécessité tout aussi universelle pour les communautés humaines d'organiser (i.e d'institutionnaliser) leurs subsistances sur la base d'une définition historiquement située des besoins. Cet oubli débouche sur deux biais constitutifs de l'erreur économiciste (Polanyi, 1947a; Polanyi, 1947b; Polanyi, 1977a). Cette erreur est en fait la confusion des deux sens possibles de l'économie:

- En ignorant les structures institutionnelles nécessaires et particulières à chaque groupe social, la théorie économique formelle en arrive à considérer implicitement l'agencement présent comme universel et en fait la pierre de touche de toute analyse. Parmi ces règles particulières universalisées, on trouve d'une part celle de la rareté, d’autre part celle de la marchandisation généralisée, illustrée dans la GT par la marchandisation de la terre, du travail et de la monnaie.

- De même qu'ils regardent les autres par eux-mêmes, les économistes voient leurs propres sociétés comme dirigées par des règles immuables auxquelles l'homme serait soumis : une «soumission volontaire et, il faut bien le dire, enthousiaste» (Polanyi, 1947b, p. 505). À ce titre, Polanyi affirme que «le système de marché a profondément déformé notre vision de l'homme et de la société», ces deux derniers semblant soumis au «déterminisme économique» (1957a, p. 521).

4 «L'institutionnalisation du procès confère à celui-ci unité et stabilité, elle crée une structure ayant une fonction déterminée dans la société; elle modifie la place du procès dans la société, donnant ainsi une signification à son histoire, elle concentre l'intérêt sur les valeurs, les motivations et la politique. Unité et stabilité, structure et fonction, histoire et politique définissent de manière opérationnelle le contenu de notre assertion selon laquelle l'économie humaine est un procès institutionnalisé» (Polanyi, 1957a, p.59). 
C'est dans l'optique d'éviter ces biais que Polanyi lance le projet substantiviste de l'étude de l'institutionnalisation des processus économiques. Il s'agit de se défaire d'une analyse économique partant du seul comportement de maximisation individuelle pour tendre vers une analyse institutionnelle. Pour Polanyi, l'individualisme pur a comme propriété de fixer analytiquement les institutions, ceci parce qu'il revient à considérer comme propre à l'humain ce qui est en réalité relatif aux institutions historiquement situées qui encadrent son action. Il faut donc accompagner l'étude de l'homme d'une étude de son environnement institutionnel. Autrement dit, là où l'économie formelle fixe la rareté et le choix comme des faits anhistoriques, l'économie substantive «implies neither choice nor insufficiency. Man's livelihood may or may not involve the need for choice. Custom and tradition, as a rule, eliminate choice, and if choice there be, it need not induced by limiting effects of any "scarcity" of means » (Polanyi, 1977b, p. 27). C’est sur ces institutions que Polanyi se propose de revenir à l'aide d'une boîte à outils conceptuels abordée en détail dans le point suivant.

\section{I.2. LES INSTITUTIONS SELON POLANYI}

Un examen des moyens par lesquels l'économie, dans le sens de la réalité économique, acquiert sa stabilité - c'est-à-dire l'interdépendance récurrente de ses membres via son institutionnalisation - passe par l'usage «d'un nombre limité de modèles que l'on pourrait appeler formes d'intégration» (1957a, p. 59). Une première taxinomie de ces formes voit le jour dans la GT. Celle-ci sera reprise et modifiée dans «L'économie en tant que procès institutionnalisé» (1957a). Dans ce dernier texte se dégagent trois formes d'intégration empiriquement observables: la réciprocité, la redistribution et l'échange. Ce triptyque concerne très précisément «le mouvement des biens, qu'il s’agisse de déplacement dans l'espace physique ou de changement de propriétaire» (Maucourant, 2005, p. 62). Chacune de ces formes d'intégration renvoie à un type d'agencement (Supporting Structure) : la symétrie, la centralité ou le marchés. Ces systèmes sont bien plus que la simple agrégation d’actes individuels : «la conduite de la réciprocité entre individus n’intègre l'économie que s'il existe des structures symétriquement ordonnées, tel

5 On trouve ici l'idée que le monde social est structuré et non réductible à son appréhension empirique. 
un système symétrique de groupes de parenté. Mais un simple comportement de réciprocité au niveau personnel ne saurait donner naissance à un système de parenté » (Polanyi, 1957a, p. 60). Ces structures sont nécessaires à la régularité des mouvements constatés à l'aide des formes d'intégration.

- La réciprocité n'est possible que s'il existe un principe de symétrie tel que Polanyi le trouve explicité dans l'œuvre de l'anthropologue Bronislaw Malinowski, Les Argonautes du Pacifique occidental. Il y est décrit un principe d'échange intertribal mélanésien prenant le nom de kula, popularisé en France par les travaux de Marcel Mauss (1924). Le principe en est, brièvement, le suivant: lors de grandes expéditions maritimes, les navigateurs opéraient à des échanges d'objets précieux, voyagu'a, selon une fréquence temporelle et un sens de rotation bien précis. Ces échanges étaient soumis à l'obligation de la réciprocité de type don-contre-don, i.e. les obligations de recevoir et de rendre: la personne qui reçoit se voit contrainte de rendre lors d'un voyage ultérieur. Dans le cadre de la kula, le mouvement réciproque des biens n'est possible que si chaque individu «possède» un correspondant dans une autre île. Autrement dit, la réciprocité ne peut s'émanciper d'une structure sociale symétrique.

- La redistribution, que Polanyi décrit comme «un mouvement d’appropriation en direction d'un centre, puis de celui-ci vers l'extérieur » (1957a, p. 53), se voit subsumée par l'instauration d'un regroupement des richesses dans une seule main, en vertu de la coutume. La société doit être structurée autour d'un centre.

- La troisième forme d'intégration est l'échange. Elle nécessite, pour être envisagée comme telle, le support d'un marché créateur de prix. Polanyi distingue trois types d'échanges: l'échange opérationnel (simple passage de main en main), l'échange décisionnel (mouvement d'appropriation à taux fixe) et l'échange intégratif (mouvement d’appropriation à taux négocié).

On peut affirmer, en gros, que tous systèmes économiques [...] étaient organisés selon les principes soit de la réciprocité ou de la redistribution, soit de l'administration domestique ${ }^{6}$, soit d'une combinaison des trois. Ces principes furent institutionnalisés à l'aide d'une organisation sociale qui utilisait, entre autres, les modèles de la symétrie, de la centralité et de l'autarcie. Dans ce cadre, la production et la distribution ordonnée des biens étaient assurées grâce au principe d'autarcie, emprunté à Aristote. Voir sur ce point Polanyi, 1957b. 
à toutes sortes de mobiles individuels disciplinés par des principes généraux de comportement. Parmi ces mobiles, le gain n'occupait pas la première place. La coutume et le droit, la magie et la religion induisaient de concert à se conformer à des règles de comportement qui lui permettaient en définitive de fonctionner dans le système économique (Polanyi, I944, p. 86).

Le point important de la grille polanyienne est que les mouvements sociaux qui s'offrent à l'observateur à l'aide des trois outils mentaux que sont la réciprocité, la redistribution et l'échange, - ces trois formes d'intégration étant des outils heuristiques et non des réalités extérieures - nécessitent des agencements institutionnels particuliers (supporting structures). À ce titre, l'action individuelle n'est possible que s'il existe une structure institutionnelle idoine. Ce mouvement conceptuel peut être qualifié d'induction à rebours en ce que Polanyi part de l'observation de la réalité des mouvements sociaux afin d'en déduire leurs conditions de possibilité, i.e. l'existence d'institutions précédant les mouvements sociaux observables. Le manque d'écrits épistémologiques, dont fait montre l’œuvre de Polanyi, interdit toute conclusion hâtive par rapport à sa méthode et au statut des concepts qu'il utilise. Les éléments rassemblés permettent néanmoins de penser le raisonnement polanyien en termes d’argument transcendantal' : étant donnée l'observation des comportements sociaux, et l'impossibilité d'expliquer ces derniers par le seul individualisme méthodologique, il ne peut en être autrement de l'existence de structures institutionnelles. On débouche alors sur une idée forte: les mouvements économiques sont intimement liés à l'ensemble des autres structures culturelles, politiques et sociales en ce que celles-ci en sont les conditions de possibilité.

Une question se pose alors quant à l'interprétation de l'œuvre de Polanyi. Dans la GT (1944), Karl Polanyi revient sur le bouleversement institutionnel ayant touché l'Europe, et plus particulièrement le Royaume-Uni, de 1795 à 1933. La postérité de cette analyse fut assurée par un concept majeur, celui de «désencastrement». On résume souvent ce dernier par l'affirmation selon laquelle cette période de l'histoire aurait été marquée par une autonomisation de la sphère marchande relativement aux structures sociales et institution-

7 Le premier à tenter d'introduire à la science économique le concept d'argument transcendantal est Tony Lawson (1997; 2003). 
nelles décrites ci-dessus. L'économie de marché serait alors assimilée à une économie désencastrée. Or, comment envisager à la fois l'économie comme processus institutionnalisé de manière incontournable et une rupture d'institutionnalisation, comme le suggèrerait l'historiographie de la GT ? Cette controverse fut initiée par Mark Granovetter (1985), pour qui Polanyi en viendrait à prôner de fait une dichotomie entre sphère économique et sphère relationnelle par l'évocation même d'un processus de désencastrement. Barber résume clairement cette proposition:

Polanyi décrit le marché comme «désencastré», les deux autres types d'échanges économiques (la réciprocité et la redistribution) n'étant plus «encastrés» dans les autres éléments socio-structurels et socio-culturels de la société. [...] Notre proposition forte, contrairement à celle de Polanyi, est que toutes les économies sont inévitablement encastrées [...] Parler de désencastrement du marché détourne notre attention de l'analyse de ce que représente cette interdépendance (1985, p. 400).

On justifie souvent ce glissement par l'aspect éminemment politique de l'œuvre de Polanyi : abandonnant le costume du théoricien pour celui du politique, il serait amené à négliger la cohérence analytique de son propos.

L’analyse de la GT que propose ce travail montre les limites de la lecture traditionnelle donnée ci-dessus. En effet, Polanyi se défend du constat d'un désencastrement de fait: «Nous ne traitons naturellement pas ici d'images de la réalité, mais de schémas conceptuels utilisés pour les besoins de la clarification. Aucune économie de marché séparée de la sphère politique n’est possible » (Polanyi, 1944, p. 259). Toutefois, force est de constater qu'à d’autres endroits, Polanyi souligne l'étroite ressemblance entre la théorie économique formelle et l'économie occidentale moderne : «Ce n’est que dans le cadre institutionnel de l'économie de marché que les lois du marché sont pertinentes » (ibid., p. 65). Ce type d'affirmation justifie le renouvellement de la critique d'une erreur polanyienne, celle d’une conception sous-socialisée des économies de marché : il existe bien un type d'économie conforme à la théorie économique formelle. Cette critique ne passe plus par le problème du désencastrement en 
tant que tel, mais par les faiblesses épistémologiques de l'auteur hongrois. C’est notamment ce que souligne Hédoin:

Selon Polanyi, l'économie formelle est applicable aux économies modernes, car il y a un parallélisme effectif entre les hypothèses de l'économie formelle et la réalité institutionnelle des économies de marché. La conception du statut épistémologique dont témoigne cette thèse est celle d'un réalisme empirique selon lequel la validité d'une théorie dépend de la proximité de ses hypothèses avec la réalité empirique étudiée et que par conséquent les concepts théoriques permettent d’accéder directement à cette réalité. De fait, il ressort de cette conception que les concepts analytiques sont plus ou moins une copie de la réalité étudiée (2009, p. 227).

Les origines de l'«erreur polanyienne» se trouvent dans le rapport fait entre phénomènes économiques et concepts utilisés par l'économiste. C'est sur ce débat qu'il faudra rebondir afin de proposer une nouvelle interprétation des travaux de Polanyi. L'objectif sera de nuancer le flottement conceptuel polanyien en tentant de l'expliquer par la démarche particulière qu'il emploie.

\section{I.3. LES FAIBLESSES ÉPISTÉMOLOGIQUES DE POLANYI}

Avant toute chose, il convient de préciser quel est le sens du terme «institution » et quel est le statut que l'on accorde à cette notion. Il existe au moins deux manières de l'envisager, deux perspectives indissociables:

- une perspective ontologique, reposant sur la question de la nature de la réalité;

- une perspective épistémologique, concernant le statut des conceptualisations utilisées et leurs relations avec l'objet de la pensée.

Dans la première perspective, l'intérêt est porté sur l'existence réelle de l'institution. Alors que dans le cadre de l'individualisme le plus pur ${ }^{8}$ (qu'il est possible de nommer ici «individualisme ontologique ») on considère que seuls les individus constituent la réalité sociale et que toute étude part irrémédiablement d'eux, une approche institutionnaliste estimerait qu'il existe, au-delà des

8 L'exemple parfait de ce point de vue reste Mises qui ne confère aucune réalité ontologique au groupe en tant qu'entité réelle, ceci sur la base de l'affirmation selon laquelle seuls les individus ont des buts et des intérêts (principe de Popper-Agassi). 
individualités, des structures qui les surpassent : groupes, sociétés, règles, traditions, coutumes, ce qu’on nomme ici de manière générique «institutions ».

La seconde perspective se penche sur l'institution comme outil de compréhension du réel. La notion d'institution renvoie alors à un simple outil conceptuel. Il est ainsi possible de considérer que ce concept a le pouvoir de nous donner un accès direct aux choses que l'on observe, ou bien qu'il existera toujours un écart irréductible entre les mots et les choses. Il convient de conserver à l'esprit cette distinction et de constamment se demander si un ensemble de propositions prétend se référer à la chose en soi (point de vue ontologique) ou au concept que l'on utilise (point de vue épistémologique). Cette pluralité de sens conduit bien souvent à produire des énoncés abscons. Il semble que Polanyi tombe régulièrement dans cet écueil, le lecteur ne sachant plus à quel niveau se situe son discours. Cette confusion est, selon la lecture proposée ici, le cœur de l'erreur polanyienne soulignée par la nouvelle sociologie économique. Afin de le comprendre, il faut démêler les deux perspectives de l'institutionnalisme (ontologique et épistémologique) dans le travail de Polanyi.

- Perspective ontologique : quelle est la nature de la réalité ? D’un point de vue ontologique, l'approche de Polanyi est réaliste. Par réalisme ontologique on entend ici toute démarche ayant comme point de départ le postulat que l'objet d'étude ultime des sciences existe toujours indépendamment (au moins en partie) des représentations que l'on s'en fait. Chez Polanyi, l'institution en tant qu'objet d'étude (i.e en tant que référent) est réelle. Comme indiqué ci-dessus, c'est parce qu'il est impossible de penser l'action individuelle sans institutions qu'est postulée l'existence réelle de ces dernières. C'est donc au nom d'un principe de causalité que les institutions sont considérées comme existantes: sans institutions, les régularités sociales seraient absentes, et aucune décision ne pourrait être prise en raison d'une incertitude fondamentale quant aux actions d'autrui. Il semble donc qu'il soit possible de classer Polanyi du côté d'une approche réaliste en ce que les concepts de groupe humain, d'institution sont censés renvoyer à des entités réelles c'est-à-dire dotées d'un pouvoir causal. 
- Perspective épistémologique ou la manière d’aborder le monde : À l'intérieur du cadre du réalisme ontologique, la perspective épistémologique fonde la distinction entre réalisme empirique et réalisme transcendantal, catégories empruntées à Lawson (I997, p. 48 ; 2003, p. 220). Ces deux positions se confrontent sur le terrain de la nature des théories utilisées pour l'étude des entités réelles du monde. Ce que Tony Lawson nomme réalisme empirique consiste en l'idée que ces théories ont la capacité d’atteindre directement les phénomènes à travers une correspondance entre ces énoncés et faits observés. Le réalisme transcendantal relève lui l'existence d'un écart irréductible entre l'objet et la connaissance de l'objet ${ }^{9}$. Sur cette distinction, Polanyi est loin d'être clair. Si, comme évoqué ci-dessus, Polanyi peut être considéré comme un partisan du second type de réalisme en ce qu'il use d'une ligne argumentative selon laquelle la simple observation des régularités sociales ne peut être une fin en soi et qu'il est nécessaire de comprendre les causalités sous-jacentes du monde social via une conceptualisation idoine construite en fonction d'un questionnement particulier, d'un autre côté, ceci paraît entrer en contradiction avec certains de ses propos. Notamment: «cette conception de l'économie comme siège de phénomènes tels que la répartition (des biens et salaires), l'épargne, l'obtention de surplus par le marché, la formation des prix, provient du milieu occidental du XVIII ${ }^{\mathrm{e}}$ siècle et est certainement judicieuse dans le cas des agencements institutionnels d'un système de marché, dès lors que les conditions réelles satisfont en gros les hypothèses fixées par le postulat d'économie» (Polanyi, Arensberg et Pearson, 1957, p. 50). Il est ici envisagé une correspondance historiquement située entre théorie économique formelle et réalité économique, c'est-à-dire la possibilité de construire une théorie conceptuellement désincarnée. C'est de cette confusion qu'émerge le débat autour de la notion de désencastrement: en évaluant la théorie économique à la lumière de sa pertinence empirique, Polanyi ouvre la porte à la possibilité d'une société désencastrée, soumise à une économie de marché, correspondant à la description de l'économie formelle.

9 «the world is composed not only of events and states of affairs and our experiences or impressions, but also of underlying structures, powers, mechanisms and tendencies that exist, wether or not detected, and govern or facilitate actuel events. 》 (Lawson, 1997, p. 21). 
L'économie de marché est donc assimilée à la théorie économique formelle réifiée en raison d'un conformisme entre réalité sociale et théorie, position révélatrice du réalisme empirique de Polanyi.

À l'encontre de cette lecture, la thèse ici défendue est que Karl Polanyi ne décrit pas une théorie réifiée, mais un lien spécifique entre les représentations des économistes et la réalité des institutions. À suivre Michel Callon, la GT «est aussi, et avant tout, une des rares tentatives de faire le lien entre science économique et économie [au sens de la réalité économique], avec une convaincante analyse du rôle de la théorie économique, telle celle de Ricardo, dans la construction d'un marché du travail» (1998, p. 2). Callon fait ainsi de Polanyi un précurseur de la théorie de la performativité des énoncés, défendant l'idée selon laquelle «La science économique, au sens large du terme, performe, modèle et formate la réalité, plutôt qu'elle n'observe la manière dont elle fonctionne» (idem). Néanmoins, cette interprétation passe à côté du problème polanyien : insister sur le lien créateur entre discours théorique et réalité n'exclut pas le problème originel, à savoir celui d'une conception désencastrée de l'économie de marché. Sauf à penser la théorie économique non plus comme un simple discours mais comme une institution forgeant les croyances individuelles. Considérons toute théorie scientifique comme une façon particulière de classer les objets observés prenant la forme d'une représentation. L'économie formelle peut ainsi être ramenée à un mode de classification des choses sur la base du présupposé de la rareté. Elle ne constitue donc pas une réalité en soi (un état de désencastrement), mais un bloc interactif: la théorie économique (abordée comme une institution), pris dans ses relations avec les autres institutions humaines. L'hypothèse posée dans la GT pour tenter de dégager les structures internes du monde social n'est pas celle d'une réification des concepts de l'économie politique formelle mais de l'existence du lock-in d'un agencement entre théories économiques et une forme particulière d'institution de l'échange. Ce blocage a pour nom désencastrement: la théorie économique est une partie de l'économie réelle, non en ce qu’elle crée ex nihilo une économie désencastrée conforme à ses modes 
de pensée, mais en ce qu'elle produit des représentations qui influencent l'évolution institutionnelle. Le concept de désencastrement est un outil de l'économie substantive servant l'étude de l'économie formelle et de ses relations avec l'ensemble de la structure institutionnelle dont le monde social est traversé. Cette relation débouche sur une complémentarité institutionnelle non-évolutive et auto-renforçante, autrement dit sur un phénomène de renforcement entre institutions économiques et théories économiques : le discours de l'économie formelle bloque l'évolution des institutions. Ce mouvement de blocage est une caractéristique essentielle des travaux de Polanyi. Ainsi, l'économie formelle n'est pas qu'une méthode théorique contre laquelle Polanyi se bat, mais également son objet d'étude principal.

Cette lecture peut paraître étriquée tant Polanyi se hâte de placer économie formelle et substantive sur un même plan épistémologique. Néanmoins, si l'écart entre théorie et réalité n’est pas souligné dans les textes principaux de Polanyi, notons qu'un de ses écrits relatifs au calcul socialiste (1922) contient une lecture moins équivoque. Il y distingue les faits économiques, les concepts comptables et la théorie économique comme trois éléments différents mais évoluant de concert : les concepts comptables sont des interprétations du monde, la théorie économique constitue une réinterprétation des concepts comptables. Les faits économiques sont donc bien séparés de leur conceptualisation par le biais d’interprétations. Par exemple : «la façon de réaliser cette vue d’ensemble [vue de l'économie réelle par la comptabilité] est toutefois différente pour chaque type d'économie, car ce que nous voulons en retirer est, à chaque fois, différent. En termes simples, la tâche de chaque système particulier de comptabilité est donc la suivante: elle doit nous fournir un éclairage chiffré sur les questions que nous nous posons à propos de cette économie» (ibid., p. 289). S’il existe une indépendance entre théorie et réalitér ${ }^{10}$, celle-ci n’est que partielle car ces deux ensembles interagissent, le monde social étant avant tout un monde de représentations influencé par les théories économiques.

10 Humphreys (1969, p. 170) souligne de plus l'attachement de Polanyi à une épistémologie nominaliste dans les sciences sociales. 
Interpréter Karl Polanyi en ces termes revient à en faire le porteur d'une perspective nouvelle considérant la science économique comme une institution. Une fois posée cette hypothèse interprétative venant nuancer les points d’achoppement épistémologiques auxquels il a été tenté de raccrocher le problème du désencastrement, il convient de voir concrètement la manière dont Polanyi suit cette route. C'est dans ce sens que la seconde partie réinterprète plus en détail la GT. Pour cela, il faudra dégager les mécanismes de la dynamique institutionnelle en action dans l'historiographie que Polanyi construit.

\section{KARL POLANYI OU L'ÉCONOMIE FORMELLE COMME INSTITUTION}

Faisant l'histoire d'un changement majeur dans le déroulement de la vie sociale, la GT dévoile la manière dont Polanyi envisage la dynamique institutionnelle. L'économie substantive, pour s'écarter de l'erreur économiciste, doit se déprendre des considérations naturalistes consistant à voir la source du changement au sein de la nature humaine elle-même. Ainsi, par exemple, là où Adam Smith voit l'origine de la division du travail dans une propension individuelle au troc $^{11}$, l'économie substantive s'attache à relever les processus historiques et institutionnels constitutifs de ce fait social. Pour ce faire, Polanyi superpose à la fois une théorie économique du changement institutionnel et une historiographie venant appuyer ses propos. Dans les deux cas, le discours de l'économiste occupe une place prépondérante : «Pour comprendre le Fascisme allemand nous devons revenir à l’Angleterre de Ricardo » (Polanyi, I944, p. 54). On reviendra, dans un premier temps, sur le récit raisonné de Polanyi, avant d'en tirer une interprétation du modèle théorique qu’il utilise.

II II est flagrant de voir que Smith, devant son incapacité à expliquer l'origine de la division du travail, fonde celle-ci sur «le penchant qui les porte [les hommes] à trafiquer, à faire des trocs et des échanges d'une chose pour une autre» (Smith, 1776, p. 8I). Comment expliquer dans ces conditions le passage d'un état sans division à une société en étant pourvue, si rien n'assure à celui qui se spécialise le respect des promesses des autres? II peut toujours arguer qu'«on a jamais vu de chien faire de propos délibéré l'échange d'un os avec un autre chien» (ibid.) afin de justifier l'absence de conventions premières à l'échange et à la division du travail, il n'en résout pas pour autant la difficulté. 


\section{I. LA GRANDE TRANSFORMATION}

Une histoire de machines, de lois et d'économistes

L'histoire de la GT est celle de la domination grandissante, mais jamais totale, de l'échange sur les autres formes d'intégration. L'échange économique (au sens marchand du terme) se serait peu à peu désencastré du reste des relations sociales pour tendre vers la constitution d'un grand Marché Autorégulateur nécessitant l'appui institutionnel de l'ensemble des structures sociales: «la maîtrise du système économique par le marché a des effets irrésistibles sur l'organisation toute entière de la société, elle signifie tout bonnement que la société est gérée en tant qu’auxiliaire du marché. Au lieu que l'économie soit encastrée dans les relations sociales, ce sont les relations sociales qui sont encastrées dans le système économique » (Polanyi, 1944, p. 88). Ainsi, s’appliquerait à l'ensemble de la société un modèle institutionnel supporté par un type unique de motivation: l'appât du gain, alimenté par l'aiguillon de la faim (nous y reviendrons). Polanyi explique cette évolution par l'émergence d'une croyance concernant le caractère à la fois naturel et inévitable de cette mutation institutionnelle, croyance diffusée par la science économique. C'est très précisément de cette mentalité de marché (1947b) que Polanyi fait l'histoire. Comme le souligne Plociniczak : «La Grande Transformation révèle, plus que l'institutionnalisation d’un Marché Autorégulé effectif, la tentative et l'échec d'institutionnalisation d'une forme de connaissance agissante socialement élaborée, mais partiellement partagée » (2007, p. 208). Cette mentalité de marché, nécessaire au bon fonctionnement d’un grand marché autorégulateur, se compose de deux types de croyances:

- une croyance relative à la motivation première de l'Homme: on construit peu à peu l'image d'un être humain gouverné uniquement par un type de rationalité de type moyens-fins dans un contexte naturel de rareté;

- des croyances relatives aux lois générales de l'économie, principalement à la constitution des salaires.

Ces deux types de croyances ont transformé l'image de la société, celle-ci étant dès à présent considérée comme un simple auxiliaire du marché. C'est ainsi que l'ensemble des objets sociaux sont peu à peu considérés comme des marchandises. Or, pour Polanyi, ces caractéristiques anthropologiques et 
institutionnelles sont avant tout une construction historiquement située que la science économique cristallise en diffusant un discours naturaliste alimentant la mentalité de marché. L'économie formelle ne devient pas réelle, elle justifie simplement la conservation d'un système institutionnel particulier dont l'origine est à chercher hors du discours scientifique: à savoir l'extension de la place de la machine dans la processus productif ${ }^{12}$.

La pénétration et la diffusion de la machine ont rendu nécessaire la production de masse afin d'optimiser l'amortissement de ces coûteuses installations. Cette nouvelle structuration industrielle force alors à substituer le mobile du gain à celui de la subsistance, en ce que produire toujours plus devient la condition même de la production: gain et subsistance deviennent synonymes (appât du gain). À ce stade est commise une première erreur théorique de généralisation ethnocentriste de la part de la théorie économique formelle: c'est en observant ce phénomène en marche qu'Adam Smith (selon Polanyi) construit la fable de l'homme naturellement porté vers le troc via sa «psychologie de marché». Est ainsi naturalisée une anthropologie pourtant (conformément à l'économie substantive) historiquement située: «des principes de comportements de ce genre ne peuvent trouver leur application que si les modèles institutionnels existants s'y prêtent » (Polanyi, 1944, p. 77). Cette erreur sera la base d'une légitimation morale de la seule recherche de l'intérêt individuel'13.

Le corollaire de la cherté de la machine, et du modèle comportemental auquel elle est propice, est l'obligation de garantir l'approvisionnement régulier en facteurs de productions, soit en travail, en terre et en monnaie. «On devait pouvoir les acheter. Il fallait donc les organiser en vue de la vente sur le marché

12 Celle-ci n'est pas à l'origine du changement, mais elle systématise un mouvement en cours: «Notre intention n'est pas d'affirmer que la machine fut la cause de ce qui arriva, mais d'insister sur le fait qu'une fois que des machines et des installations complexes avaient été utilisées en vue de la production dans une société commerciale, l'idée d'un marché autorégulateur ne pouvait que prendre forme» (Polanyi, 1944, p.68).

13 «Les savant proclamaient à l'unisson que l'on avait découvert une science qui ne laissait pas le moindre doute sur les lois qui gouvernaient le monde des hommes. Ce fut sous l'autorité de ces lois que la compassion fut ôtée des cœurs et qu'une détermination stoïque à renoncer à la solidarité humaine au nom du plus grand bonheur du plus grand nombre acquis la dignité d'une religion séculière» (Polanyi, 1944, p. 144). 
- en d'autres termes comme marchandises» (ibid., p. II I). Pour ce qui est du travail, cette «marchandisation » se fit en deux étapes juridiques ${ }^{14}$. La première fut la mise en place des lois sur les enclosures, qui jetèrent les fermiers nécessiteux hors de la pratique agricole et permirent la création d'un prolétariat rural de masse. L'obligation de clôturer les parcelles ne put être respectée de tous en raison du coût de tels travaux, ce qui conduisit à une redistribution des terres ainsi qu'à un renvoi de leurs ex-propriétaires sur le marché du travail. La seconde étape fût le Poor Law Amendment Bill de i834, selon Polanyi le point de départ véritable du capitalisme moderne. Il met fin à la loi du Speenhamland, qui garantissait à chacun un revenu de subsistance. De cette manière, l’armée de réserve suscitée par la loi sur les enclosures est désormais dans l'obligation vitale de se proposer aux portes des usines (aiguillon de la faim), garantissant aux producteurs une mise à disposition continue de la main d'œuvre.

On a jusqu'ici décrit la manière dont un choc, l'introduction de la machine, a influencé I) les comportements individuels et les hypothèses habituelles quant aux conditions de survie (il faut produire plus, il faut offrir son travail à l'industrie naissante) ; 2) le contenu de la théorie économique (la généralisation théorique d'un fait particulier) ; 3) les institutions formelles (la mise en place de nouvelles lois). Conformément à la problématique ici traitée, il convient de se concentrer sur le mécanisme de rétroaction entre science économique et structures institutionnelles. En effet, si les réformes se mettent en place, c'est en partie grâce à la médiation de la théorie économique qui tourne son regard vers la société :

L'esprit de l'homme, saisi d'une inquiétude nouvelle, se tourna vers sa propre communauté $[. .$.$] les hommes dirigèrent leurs regards sur leur propre collectif,$ comme s'ils en avaient auparavant négligé la présence. Un monde fut découvert dont on n'avait pas même suspecté l'existence, celui des lois qui gouvernent une société complexe (Polanyi, 1944, p. 12I).

14 Notre lecture sera naturellement tronquée. Nous nous concentrerons ici sur le cas du travail. De plus, nous passerons outre l'argumentation de Polanyi concernant le désenclavement des mécanismes de marché créateur de prix agissant au sein des ports et des bourses de commerce locaux. 
Ce regard, que constitue la théorie économique classique, prend forme en Angleterre durant la période pré-Poor Law Amendment Bill, celle du Speenhamland. Entre 1795 et I834 est mis en place un barème de complément salarial indexé sur le prix du pain. Ce barème eut comme effet réel, toujours selon Karl Polanyi, de faire tomber les salaires jusquau niveau de subsistance, car «personne ne travaillerait pour un salaire s'il pouvait gagner sa vie sans rien faire» (ibid., p. II5) dans le cadre d'un système où le salaire dépasse rarement ce même minimum de subsistance assuré par Speenhamland. À ce titre, «En l'espace de quelques années, la productivité du travail se mit à baisser au niveau de celle des indigents, ce qui fournit aux employeurs une raison supplémentaire pour ne pas augmenter les salaires au-delà de ce que fixait le barème » (idem). La seconde erreur économiciste, après celle de la naturalisation de l'appât du gain, fut de considérer la stagnation des salaires au niveau de subsistance comme le résultat de lois économiques naturelles, respectivement la loi des rendements décroissants et la loi de population ${ }^{15}$, alors même que «l'élément naturaliste des fondements de l'économie orthodoxe est la conséquence des conditions créées en premier lieu par le système de Speenhamland» (ibid., p. I7o). La découverte des lois sociales a été le facteur accélérant de la mutation en marche par le biais du réformisme (Poor Law Amendment Bill). Si chez Smith, nous dit Polanyi, la sphère économique n'était pas soumise à des lois propres, son fonctionnement étant en accord avec la nature humaine, pour une nouvelle génération de penseurs sociaux (Townsend, Ricardo, Malthus) les lois extérieures et naturelles s'imposent à l'Homme: «Par quelle loi l'ouvrier est-il destiné à obéir à un maître auquel il n’est lié par aucun lien légal? Quelle est la force qui sépare les classes de la société comme si elles étaient des espèces différentes d'êtres humains? et qu'est-ce qui maintient l'équilibre et l'ordre dans cette collectivité humaine qui n'invoque pas, ne tolère pas même l'intervention du gouvernement politique?» (ibid., p. I60), voici les questions en jeu. Pour y répondre, on convoque les lois économiques précitées. C’est au nom de celles-ci que Ricardo et Malthus se prononcent pour l'abolition des réglementations sur

15 «Dans les deux cas, les forces en jeu étaient les forces de la Nature, l'instinct sexuel des animaux et la croissance de la végétation dans un sol donné» (Polanyi, 1944, p. 172). 
les pauvres ${ }^{16}$. «Le libéralisme économique, dont l'intérêt était purement académique, s'enhardit et devient d'un activisme sans borne » (ibid., p. I87). Pour les classiques (Malthus et Ricardo), la situation sociale désastreuse qui accompagne cette période de l'histoire (venant contredire la proposition smithienne selon laquelle l'abondance se répercute sur le peuple) est le fruit de la conjonction entre une mauvaise loi (Speenhamland) et une tendance naturelle de l'économie à ramener les salaires à un niveau de subsistance. D’une part les salaires se maintiennent à un niveau de subsistance, il ne saurait en être autrement, d'autre part Spennhamland serait un non sens en ce qu'elle «désinciterait» au travail et entraverait purement et simplement l'essor naturel de l'économie de marché. Mais, si pour ces auteurs il convient de réformer en tenant compte des lois naturelles de l'économie ainsi que d'un certain but socialement défini (par exemple, le plus grand bonheur pour le plus grand nombre ${ }^{17}$ ), il en est tout autrement pour Joseph Townsend qui pousse au plus loin le cynisme économique dans sa Dissertation on the Poor Laws: «La faim apprivoisera les animaux les plus féroces, elle apprendra la décence et la civilité [...] la faim seul peut éperonner et aiguiller [les pauvres] pour les faire travailler, et pourtant nos lois ont dit qu’ils ne doivent jamais avoir faim» (cité par Polanyi, 1944, p. 158). À partir de I840, toujours selon Polanyi, l’activisme cesse d’être dirigé par le désir d’améliorer la situation socioéconomique pour s'intégrer au sein d’une véritable foi. Toute entrave aux «lois » du marché est contre-productive et nuisible :

On croit désormais que le marché autorégulateur découle de lois inexorables et de la Nature et qu'il est d'une nécessité inéluctable que le marché soit libéré, qu'il soit débarrassé de toute entrave. La création d'un marché du travail est un acte de vivisection pratiqué sur le corps de la société par ceux qui se sont

16 «Les lois de la gravitation ne sont pas plus certaines que ne l'est la tendance qu'auraient de pareilles lois [lois sur les pauvres] à changer la richesse et la puissance en misère et en faiblesse, en faisant renoncer l'homme à tout travail qui n'aurait pas pour unique but celui de se procurer des subsistances. (Ricardo, 1817, p. 93).

17 Notons, en effet, que, comme chez Bentham, pour Ricardo, Smith ou Malthus, le libéralisme n'est pas une fin en soi. II convient d'en étudier les effets bénéfiques au cas par cas. II peut arriver qu'en certaines situations, les mécanismes du marché ne soient pas efficients en vu de principes éthiques prédéfinis. Cette démarche diffère considérablement de celle de Spencer, pour qui les mécanismes spontanés sont considérés comme étant tout le temps les meilleurs moyens pour atteindre l'harmonie sociale. Sur ce point, voir Vergara, 2002, p. 33-36. 
endurcis à la tâche grâce à l'assurance que seule la science peut donner. Les lois sur les pauvres doivent disparaître (ibid., p. 174).

L’idée première de Polanyi est donc que les économistes classiques ont renvoyé à la société une vision biaisée en se laissant piéger par le système institutionnel de Speenhamland: appât du gain, loi des rendements décroissants et loi de la population, bien qu'étant de concepts explicatifs forgés dans et pour un système institutionnel précis, sont naturalisés et agissent en retour sur l'évolution institutionnelle via le réformisme social d'une part, la diffusion d’une mentalité de marché de l'autre. Pour Polanyi, Speenhamland «a donné l’apparence d'une économie de marché à ce qui était en réalité le capitalisme sans marché du travail» (ibid., p. I7I).

Parallèlement à ce mouvement de transformation, structuré et amplifié par la théorie économique, Polanyi identifie un contre-mouvement basé sur une dynamique similaire. Les nouvelles structures institutionnelles destinées à répondre au stimulus de la machine vont créer de nouvelles conditions de vie entraînant une réaction de la part des populations. Car si le travail est transformé en marchandise, celle-ci n'est que fictive en ce qu'on ne peut la manipuler impunément sans affecter l'homme qui en est le porteur:

Permettre au mécanisme du marché de diriger seul le sort des êtres humains et de leur milieu naturel, et même, en fait, du montant et de l'utilisation du pouvoir d’achat, cela aurait pour résultat de détruire la société. Car la prétendue marchandise qui a pour nom «force de travail» ne peut être bousculée, employée à tort et à travers, ou même laissée inutilisée, sans que soit également affecté l'individu humain qui se trouve être le porteur de cette marchandise particulière» (ibid., p. I08).

L'homme étant bousculé, on voit émerger au Royaume-Uni un certain nombre de contre-forces. En I784, alors que les conditions du travail industriel se dégradent, David Dale crée l'usine de New Lanark en Écosse, fabrique de coton autour de laquelle prend place un village aux conditions de vie « décentes » (arrêt de la journée de travail à I9h, écoles pour les enfants etc.). De nombreuses communautés fleurissent sur le même modèle. La volonté ouvrière se matérialise elle par une forte poussée syndicaliste. Le i7 juin I779, devant l’ampleur de la situation, William Pitt premier ministre anglais, fait alors voter une loi inter- 
disant toute forme de coalition. Celle-ci sera à l'origine de nombreuses rébellions ouvrières, dont le luddisme reste l'épisode le plus célèbre, jusqu’en I825 et le rétablissement du droit d'association. C'est alors que un certain Robert Owen crée le Great Consolidated Trade Union, se proposant de regrouper un certain nombre de syndicats déjà existants. «À l'origine, le mouvement owénien n’était ni un mouvement politique ni un mouvement ouvrier. Il représentait les aspirations des gens du peuple, frappés par l'avènement de l'usine, qui voulaient découvrir une forme d'existence qui ferait de l'homme le maître de la machine » (Polanyi, 1944, p. 225). Ce mouvement connaîtra un succès sans précédent (plus de 500 o०० adhérents), puis une violente répression avant de se réincarner dans la Charte du Peuple en 1838. Le mécontentement social monte donc de l'individu bousculé et se matérialise en structures institutionnelles (syndicats, communautés etc.). Mais, et ce point ressort particulièrement dans les textes plus tardifs de Polanyi, (1947b; 1977a), le mythe de la possibilité d'un marché autorégulateur ne prend jamais véritablement fin, essentiellement en raison de la persistance d'une mentalité de marché, relayée par la domination de l'économie formelle au sein de la structure théorique.

Pour en revenir au marché autorégulateur, il a été le fruit de la rencontre entre un état spécifique de l'histoire européenne et une mentalité relayée par les théories sociales. Comme le rappelle Maucourant, la grande thèse du récit polanyien est «le rôle structurant des représentations dans la fabrique de la société» (2005, p. I30). Les représentations collectives ont été un moteur du mouvement engagé au milieu du XIX ${ }^{\mathrm{e}}$ siècle, le vecteur performatif des idéologies libérales, ce qui faisait dire à Hutchison en 1851 «Nous sommes dirigés par les philosophes et les économistes» (cité par Maucourant, 2005, p. I30). Pour Polanyi, le terme de la construction d'un grand marché se manifeste par l'apogée du contre mouvement avec quatre grands évènements: le New Deal, le Front populaire, le soviétisme et le fascisme, qui induisent de nouveaux rapports institutionnels : «inévitablement, la société prit des mesures pour se protéger, mais toutes ces mesures, quelles qu'elles fussent, compromirent l'autorégulation du marché, désorganisèrent la vie industrielle, et exposèrent ainsi la société à d’autres dangers » (Polanyi, 1944, p. 22). 
L'historiographie polanyienne intègre donc de manière patente le discours économique comme facteur d'évolution institutionnelle, en jouant à la fois sur les mentalités et sur les structures juridiques.

\subsection{UNE RECONSTRUCTION DE L'HISTORIOGRAPHIE POLANYIENNE}

L’observation localisée d'un changement institutionnel permet de dégager le rôle de la science économique au sein d'une structure théorique unifiée. Pour être plus précis, le présent travail identifie dans l'historiographie polanyienne une conceptualisation du monde social en ensembles, en blocs distincts et interconnectés permettant de comprendre la réalité sociale comme un ensemble structuré d'éléments interdépendants. Ces blocs, et les relations qui les unissent serviront à éclaircir les mécanismes causaux à l'œuvre chez Polanyi. Ils sont au nombre de trois: un bloc de l'action, un bloc formel, un bloc théorique. L'articulation de ces blocs sera la base d'une reconstruction rationnelle qui permettra à la fois de réinterpréter l'écrit principal de Polanyi dans le sens de la notion de performativité des énoncés théoriques, mais également de généraliser le raisonnement polanyien en un ensemble conceptuel utile à l'explication et à la compréhension du phénomène de la performativité au-delà de l'épisode historique que l'auteur prend en considération.

- Le bloc de l'action: Il constitue le niveau de l'action individuelle socialisée. L'institution, en tant que règle, nécessite un niveau de croyance, i.e une certaine institutionnalisation des esprits, ceci afin que la règle atteigne un niveau d'auto-exécution suffisant pour ne pas être remise en cause. En d'autres termes, le niveau d'opérationnalité du bloc formel dépend en partie du degré de croyance des individus. Cette croyance se fonde sur l'expérience quotidienne, elle peut être influencée directement par le bloc théorique, via l'éducation ou tout autre média, et/ou par un processus d'essais-erreurs induit par le cheminement de l'individu dans le monde. Ce monde peut être celui de la nature ou celui de l'institution formelle qui se définit elle-même par ses règles constitutives $^{18}$. C'est au niveau du bloc de l'action qu'est placé le principe d'imaginaire

I8 La notion de règle constitutive est empruntée à John Searle Par opposition aux règles régulatives, qui encadrent une activité déjà existante, les règles constitutives créent la possibilité 
social. Par imaginaire social, on entend une croyance collective tacite, en ce qu'elle semble naturelle à celui qui la soutient.

- Le bloc formel : Niveau de la règle formelle en tant que résultat des constructions humaines. Ces règles peuvent provenir du bloc de l'action, en ce qu'est rendu formel ce qui ne l'était pas (la coutume, les pratiques informelles etc.). Une règle peut alors répondre à un besoin défendu par les individus par le biais, pour reprendre l'expression de Commons, de transaction de réparti$\operatorname{tion}^{19}$ : la lutte pour un ordre particulier. L'établissement de ces règles passe également par le bloc théorique, essentiellement par le rôle d’ingénieur social qu'endosse l'homme de science.

- Le bloc théorique: Ce bloc est le point central de l'argumentation. Il fait office de média en ce qu'il observe, théorise et restitue le monde qu'il observe et diffuse ses théorisations aux niveaux du bloc formel et du bloc de l'action en investissant les consciences collectives (ceci via des formes diverses, on pense naturellement à l'éducation mais ce lien est loin d'être le seul). La science crée des concepts à travers lesquels les agents envisagent le monde. Cette vision, nécessairement biaisée par la conceptualisation scientifique, sert de base à leurs anticipations.

Cette typologie ${ }^{20}$ est à saisir au sens d'un homomorphisme structurel entre les différents niveaux de la structure sociale (société, groupe, entreprise, famille etc.). Par exemple, le processus d'essai/erreur peut très bien saisir les actions-réactions des institutions formelles agissantes. De plus, notons que ces blocs ne constituent que des idéaux-types. Les frontières qui les séparent sont donc poreuses et variables en fonction du point de vue de l’observant, d'où cet homomorphisme structurel. Cette interprétation de la

d'une activité. L'exemple le plus courant est celui d'un jeu: il existe par ses règles, et ne peut évoluer indépendant d'elles.

19 Par transaction de répartition, J. R Commons désigne les transactions de répartition des bénéfices ou pertes de l'activité entre les membres. Elles engagent d'un côté des agents ayant autorité dans ce partage, de l'autre les différents membres participant à cette activité. On y lutte pour le pouvoir et l'ordre.

20 Cette typologie n'est pas sans rappeler la fameuse distinction poppérienne des trois mondes, notamment entre le second monde, celui des «états de conscience, ou des états mentaux, ou peut-être des dispositions comportementales à l'actions» (Popper, 1972, p. I8I), et le troisième monde, «le monde des contenus objectifs de pensée, qui est surtout le monde de la pensée scientifique, de la pensée poétique et des œuvres d'art» (ibid., p. |8|-|82). 
structure théorique implicite des travaux de Polanyi permet de réinterpréter sa démarche historico-narrative connexe. Elle constitue en quelque sorte ce que Bruce Caldwell appelle un basic economic reasoning (2002) : un moyen heuristique simple, servant à l'interprétation du monde. Le cas est ici particulier en ce que ce modèle est utilisé pour lire les travaux de Polanyi, ceux-ci étant eux-mêmes une lecture particulière du monde.

Reprenons l'histoire de la GT en utilisant cette conceptualisation en trois blocs. La première étape de l'histoire est l'apparition et la diffusion de la machine au sein de l'appareil productif (sur la Figure I : mouvement I). Cet événement vient perturber les habitudes des individus en ce que la règle de survie en place se trouve inopérante (processus d'essai/erreur). Cette gêne donne lieu à une demande de changement institutionnel : les producteurs ont besoin de sécuriser leurs entrées de facteurs de production $(2$ : transaction de répartition). La règle change, les lois sur les pauvres sont abrogées (3). La science économique forge son regard sur le monde pré-Poor Law Amendment Bill (4), elle en tire les conclusions théoriques que l'on sait (loi des rendements décroissants et loi de population). Ce raisonnement théorique donne lieu à des recommandations politiques (Malthus et Ricardo soutiennent l'amendement), ainsi qu’à un changement de représentations sociales (5) entraînant une légitimation de l'agencement institutionnel en place. C'est le sens de la citation polanyienne située en épigraphe de cet article:

Choisissez, par exemple, une motivation quelconque, et organisez la production de manière qu'elle incite effectivement les individus à produire; vous aurez, en même temps, créé une image de l'Homme telle qu'il semblera totalement réduit à cette seule motivation. [...] La motivation choisie dessinera la figure de l’Homme réel (Polanyi, I977a, p. I8). 
Figure I : le pattern model polanyien ${ }^{21}$

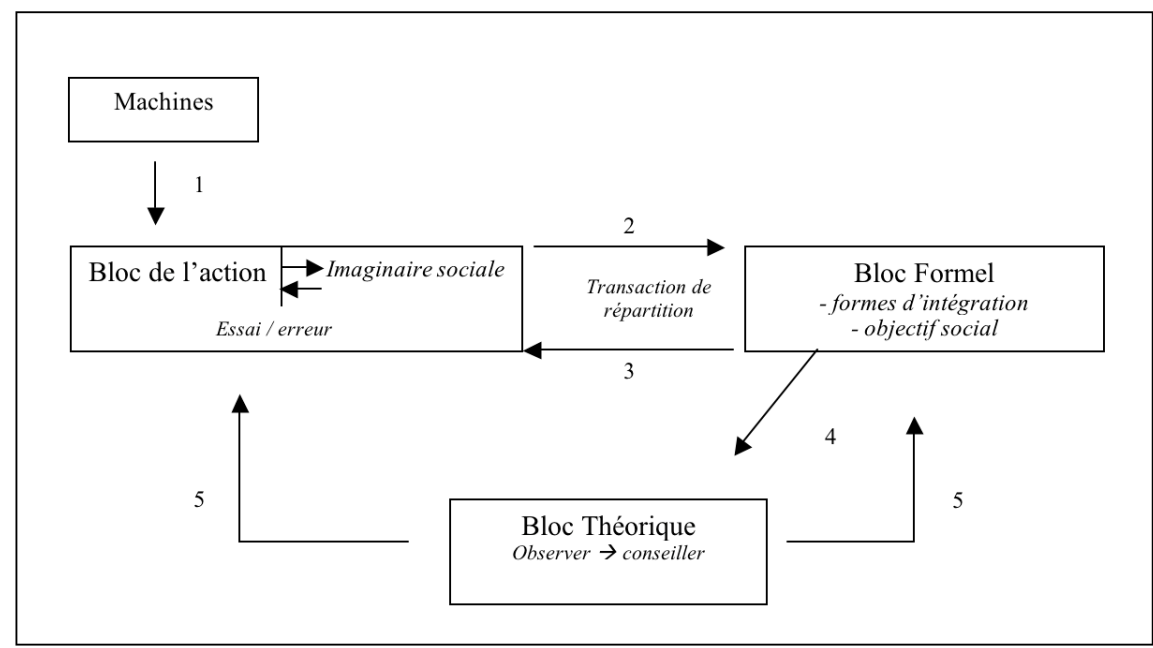

Du point de vue de l'économie substantive, le discours économique vient légitimer une évolution vers un agencement de formes d'intégrations et de supporting structure, i.e. échange et marché, ceci en renvoyant une image biaisée de la société, c'est-à-dire à la fois au niveau du bloc de l'action et du bloc formel ${ }^{22}$. Ainsi, l’idée d'un homme guidé uniquement par des considérations matérielles fut naturalisée et diffusée par les sciences économiques :

C’est cet utilitarisme pratique, ainsi généralisé, qui a forgé, de manière décisive, la compréhension qu’a l’homme occidental de lui-même et de sa société. En ce qui concerne l’Homme, nous fûmes amenés à accepter l'idée que ses motivations sont soit «matérielles» soit «idéales» et que les forces qui contribuent à l'organisation de la vie quotidienne dérivent nécessairement des motivations matérielles (idem).

21 Cette figure se fait selon notre propre reconstruction rationnelle de l'historiographie polanyienne, prenant la forme de la distinction entre les trois blocs.

22 Sur ce point, notons que l'argumentation polanyienne évite la question, pourtant essentielle, de la justification d'une influence unique de l'économiste sur le monde social. À ce titre, il est ici clair qu'il ne règle pas le problème de la discrimination entre les théories et idéologies «disponibles» dans le champ social. 
S’est alors développée la croyance en la possibilité d’une société dirigée entièrement par des motifs économiques, une société gérée en tant qu'auxiliaire du marché : «c'est ainsi que l'erreur a consisté à poser une équivalence entre l'économie humaine en général et sa forme marchande» (ibid., p. I2).

Cette fonction du bloc théorique ne serait pas en soi un problème pour Polanyi si l'erreur économiciste, se traduisant par une inertie institutionnelle, n’avait pas vu le jour: la science économique, en diffusant une image d'un ajustement institutionnel donné, ralentit le processus d'évolution ${ }^{23}$. Une question survient naturellement: sommes-nous toujours dans une société de marché caractérisée par la croyance en un grand marché autorégulateur? La réponse apportée par la GT semble être négative: "Après un siècle d'“amélioration aveugle", l'homme restaure son "habitation” » (Polanyi, 1944, p. 322). Néanmoins, cela est moins clair dans ses textes ultérieurs. Le schéma interprétatif en termes de dynamique institutionnelle, incorporant la science économique elle-même, permet de nuancer cette réponse. Si la transformation décrite par Polanyi n'est plus un état de désencastrement ou d'encastrement de fait, mais une dynamique de mystification du réel par la science économique, l'ajustement institutionnel peut se modifier et l'idée d'un marché autorégulateur rester latente, essentiellement par l'effet du discours économique. C’est le sens des deux violentes catilinaires que constituèrent «our obsolete market mentality» (1947b) et «la fallace de l'économisme» (1977a), ainsi que des trois thèses de «Faut-il croire au déterminisme économique?» (1947a) : I) le déterminisme est une invention propre au XIX ${ }^{\mathrm{e}}$ siècle; 2) les structures institutionnelles formelles ont déformé notre vision de l'homme et de la société ; 3) ces conceptions sont maintenant un obstacle à l'évolution institutionnelle de notre civilisation. Cet effet d'hystérèse de la croyance est endogène à la structure institutionnelle en ce que la science économique, comme partie intégrante de cette structure, est perçue comme une science des lois économiques naturelles du monde des hommes. Au-delà de l’interprétation historique, le récit polanyien fournit le détail des mécanismes à l’œuvre dans le phénomène de la performativité. 


\section{CONCLUSION}

Les travaux de Karl Polanyi débouchent sur une critique de la prétendue objectivité de la science économique et de la séparation entre économie et politique. Le mouvement de désencastrement n'est pas celui des structures économiques mais de la science économique vis-à-vis du politique, débouchant sur un blocage de l'évolution institutionnelle. Ainsi interprété, Karl Polanyi ouvre une perspective intéressante: celle de l'intégration, à la science économique, de la notion de performativité des énoncés théoriques, par le biais d'une étude de la dynamique institutionnelle. Une telle relecture permet à la fois de nuancer les critiques de la thèse du désencastrement et ouvre une perspective nouvelle à l'institutionnalisme en intégrant la science économique comme institution à part entière.

La science économique, via la diffusion de croyances, tend à légitimer le mode de pensée dont elle est porteuse et influence le monde réel dans ce sens. Voici en substance le discours de Polanyi concernant l'économie formelle. Trop d'interprétations de la GT se focalisent sur le récit de la construction sociale d'un grand marché autorégulé. Réduite à cela, elle n’a qu’un moindre intérêt, qui doute encore que le marché soit une construction humaine et politique? Comme le souligne Ian Hacking (1999), la rhétorique du constructivisme n’a d'intérêt qu'à la lumière d’un dévoilement plus profond : celui de l'inévitabilité. Dire que «X» est socialement construit, sans tomber dans la banalité, c'est soutenir trois choses: (I) X n'est pas inévitable, (2) X, tel qu'il est, est médiocre, (3) le monde irait mieux sans X. Tout cela n'a toutefois aucune valeur sans l'affirmation préalable: (o) X est tenu pour inévitable (Hacking, 1999, p. 37-38). Dans le cas polanyien, le raisonnement se fait sur deux niveaux. À un premier niveau, $\mathrm{X}$ est une société de marché telle que les économistes classiques pensent l'observer en puissance. À un second niveau, X est la croyance en l'inévitabilité des forces économiques à l'œuvre dans le monde. Cette croyance est portée par les individus qui, confrontés aux difficultés, se résignent à la situation: ils supportent l'institution en place sans la considérer nécessairement comme bonne mais uniquement comme inévitable. 
L’œuvre de Polanyi est tout entière tournée vers le dévoilement de ces croyances à l'origine d'un renforcement des institutions en place. Quelle forme prend ce dévoilement? Polanyi décrit un mouvement de blocage de l'évolution sur la base d'une tendance des structures en place à se légitimer par le biais du discours scientifique. Cette légitimation passe par la diffusion de croyances dont l'ensemble forme la mentalité de marché. Celle-ci vient faciliter l'introduction de la machine au sein de l'appareil productif. Cette mutation technologique rend en retour audible le discours performatif des économistes par une partie de la population intéressée par la mise en place d’un marché autorégulateur. Polanyi semble ici influencé par les travaux du jeune Marx sous la plume duquel on lit:

On comprend donc que seule l'économie politique, qui, avec Adam Smith, a reconnu le travail comme son principe et a cessé de voir dans la propriété un simple état de choses extérieur à l'homme, doit être considérée, d'une part, comme le produit de l'industrie moderne, et, d'autre part, comme une force qui a elle-même accéléré et célébré le dynamisme et le développement de cette industrie et en a fait une force de la conscience (I844, p. I35).

Cette influence passe principalement par deux biais : l'ingénierie sociale et les représentations des agents. Il est possible de résumer ainsi le raisonnement polanyien: d’une part, l'histoire compte et la théorie économique doit suivre cette histoire sans poser les concepts qu'elle invoque comme immuables; d’autre part, l'économiste influence le monde social qu'il décrit. C’est cette même intuition qui frappera Popper (1982, p. I06) lorsqu'il soulignera, bien plus tard, «la nature incomplète d'un univers qui contient les objets du savoir ».

NdA. Une version précédente du présent travail a été présentée lors du XIIle Colloque International de L'association Charles Gide pour l'Étude de la Pensée Économique. Je remercie Laure Bazzoli et Véronique Dutraive qui m'ont fait le plaisir de stimulants rapports. Je remercie Roberto Baranzini, Pascal Bridel, Jérôme Lallement et Jérôme Maucourant pour nos discussions et leurs précieux commentaires. Je remercie également les membres du séminaire interne du centre Walras Pareto, ainsi que ceux du séminaire $\mathrm{AOH}$. Enfin, je tiens à remercier Marine Bonnard, Hervé Guillemin et Cyril Hédoin pour leurs commentaires concernant ce texte ou des versions précédentes. Je reste évidemment seul responsable des erreurs subsistant dans ce texte, ainsi que des interprétations qu'il défend. 


\section{BIBLIOGRAPHIE}

AOKI M., 200I, Fondements d'une analyse institutionnelle comparée, Albin Michel, Paris.

AUSTIN J. L., 2006 (1962), Quand dire c'est faire, Paris, Seuil.

BARBER B., 1995, «All economics are 'embedded': the career of a concept on beyond», Social Research, 62-2, p. 387-4I4.

BLOCK F., 2003, «Karl Polanyi and the writing of The Great Transformation», Theory and Society, 32-3, p. 275-306.

BRISSET N., 20II, «Les limites de la performativité des énoncés en économie: les apports de John Searle», Economia, I-4, p. 557-588.

CALDWELL B., 2002, «In Defense of Basic Economic Reasoning», Post-autistic review, I3.

CALLON M., 1998, The Laws of the Markets, Oxford, Blackwell.

CHAVANCE B., 2007, L'Économie institutionnelle, Paris, La Découverte.

DENIS J., 2006, «Les nouveaux visages de la performativité», Études et communication, 29, p. 7-24.

DUMEZ H. et JEUNEMAîTRE A., 2005, «La démarche narrative en économie», Revue économique, 56-4, p. 983-1005.

GRANOVETTER M., 1985, «Economic Action and Social Structure: The Problem of Embeddedness», The American Journal of Sociology, 9I-3, p.485-5I0.

HACKING I., 2008 (1999), Entre science et réalité, la construction sociale de quoi?, Paris, La Découverte.

HAYEK F. A., 2007 (1967), «Le résultat de l'action humaine mais non d'un dessein humain», in. F. A. Hayek, Essais de philosophie, de science politique et d'économie, Paris, Les Belles Lettres, p. 159-172.

HÉDOIN C., 2009, Le rapport entre théorie et histoire dans les approches historicistes et institutionnalistes en économie: essai de reconstruction rationnelle du programme de recherche scientifique de l'institutionnalisme historique, Thèse de doctorat, Université de Reims Champagne-Ardenne, Reims.

HODGSON G.M., 2000, «What Is the Essence of Institutional Economics», Journal of Economic Issues, 34-2, p. 317-329. 
-, 2002, «The Evolution of Institutions: An Agenda for Futute Theoretical Research», Constitutional Political Economy, 13, p. III-27.

HUME D., 1993 (1740), Traité de la nature humaine: Les passions, Paris , Flammarion. HUMPHREYS S. C., 1969, «History, Economics, and Anthropology: The Work of Karl Polanyi», History and Theory, 8-2, p. 165-212.

LAWSON T., 1997, Economics and Reality, New York, London, Routledge.

-, 2003, Reorienting Economics, New York, Routledge.

LE VELLY R., 2007, «Le problème du désencastrement», La revue du Mauss, 29, p. $181-196$.

MACKENZIE D., MUNIESA F. et SIU L. (eds), 2007, Do Economists Make Markets?, Princeton, Princeton University Press.

MALINOWSKI B., 1989 (1922), Les Argonautes du pacifique occidental, Paris, Gallimard.

MARX K. 1996 (I844), Manuscrit de 1844, Flammarion, Paris.

MAUCOURANT J., 2005, Avez-vous lu Polanyi?, Paris, La Dispute.

- et PLOCINICZAK S., 2009, «L'institution selon Karl Polanyi. Fondements et mise en perspective contemporaine», Tracés, 17-2, p. 143-I56.

MAUSS M., 2007 (1924), Essai sur le don, Paris, PUF.

ORLÉAN A., 1989, «Pour une approche cognitive des conventions économiques», Revue économique, 40-2, p. 241-272.

PLOCINICZAK S., 2007, «Au-delà d'une certaine lecture standard de la Grande Transformation», Revue économique, 29, p. 207-224.

POLANYI K., 1983 (1944), La Grande Transformation, Paris, Gallimard.

-, 1986 (1977a), «La fallace de l'économisme», Bulletin du MAUSS, I8, p. II-26.

-, 1977b, The Livelihood of Man, New York, San Fransisco, Londres, Academic Press.

-, 2008, Essais de Karl Polanyi, sous la direction de Michele Cangiani et Jérôme Maucourant, Paris, Seuil.

- - (1922), «La comptabilité socialiste», p. 283-315

- - (1947a), «Faut-il croire au déterminisme économique», p. 521-528. 
- - (1947b), «La mentalité de marché est obsolète», p. 505-528.

- - (1957a), «L'économie en tant que procès institutionnalisé p.53-77.

- - (1957b), «Aristote découvre l'économie», p.53-77.

- - ARENSBERG C.M. et PEARSON H.W., (1957), «La place de l'économie dans les sociétés », p.49-52.

POPPER K., 1991 (1972), La Connaissance objecive, Paris, Aubier.

-, 1984 (1982), L'Univers irrésolu, Paris, Hermann.

RICARDO D., 1977 (1817), Des principes de l'économie politique et de l'impôt,

Paris, Flammarion.

ROBBINS L., 1947 (1932), Essai sur la nature et la signification de la science économique, Paris, Librairie de Médicis, 1947.

SEARLE J. R., (2005), «What is institution?», Journal of Institutional Economics, I-I, p. I-22. -, 1998 (1995), La Construction de la réalité sociale, Paris, Gallimard.

SMITH A. 199I (1776), Recherche sur la nature et les causes de la richesse des nations, Tome I, Paris, Flammarion.

VERGARA F., 2002, Les Fondements philosophiques du libéralisme, Paris, La Découverte. WEBER M., 1996 (1910-1920), Sociologie des religions, Paris, Gallimard, 1996.

ZELIZER V., 1994, The Social Meaning of Money. Pin Money, Paycheks, Poor Relief, and Other Currencies, New York, Basic Books. 
